\title{
Avaliação de tecnologias em hospitais de ensino: desafios atuais.
}

Technology assessment in teaching hospitals: current challenges.

Evaluacíon de tecnologías sanitarias: retos actuales.

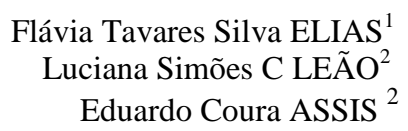

RESUMO: Objetivo: Analisar as características estruturais e processos de trabalho após dois anos da implementação de centros de avaliação da tecnologia em hospitais de ensino no Brasil. Método: Este estudo utilizou um questionário auto administrado online que pesquisou gerentes de vinte e quatro centros que participaram do concurso público 2009. Resultados: um total de Vinte gerentes concluíram o inquérito. Quatorze centros responderam que houve a formalização da área para o hospital. Todos eles relataram dificuldades em pessoal qualificado e recursos financeiros, as equipes foram capazes de oferecer cursos (dezesseis centros) e produzir algumas avaliações como os pareceres científicos (doze centros). Dezenove adotaram as orientações metodológicas de REBRATS. Conclusão: Percebeu-se que dois anos de execução destes centros não foram suficientes para atingir as características estruturais e processuais considerando $o$ levantamento. A difusão da cultura HTA em um país sem tradição como o Brasil deve ser uma ação permanente para a qualidade dos cuidados de saúde.

Palavras-chave: Avaliação da Tecnologia Biomédica, hospitais

\begin{abstract}
Objective: To analyze the structural characteristics and work processes after two years of the implementation of assessment centers of technology in teaching hospitals in Brazil. Method: This study used a online self-administered questionnaire that surveyed managers of the twenty-four centers that attended of the public call 2009. Results: a total of twenty managers completed the survey. Fourteen centers answered that occurred the formalization of the area into the hospital. All of them reported difficulties in trained personnel and financial resources, the teams were able to offer courses (sixteen centers) and produce some assessments as the scientific expert advice (twelve centers). Nineteen adopted the methodological guidelines of REBRATS. Conclusion: It was realized that two years of implementation of this centers were not enough for achieve structural and procedural characteristics considering the survey. The dissemination of HTA culture in a country with no tradition like Brazil should be a permanent action for quality of healthcare.
\end{abstract}

\footnotetext{
${ }^{1}$ Pesquisador em saúde pública na Fiocruz Brasília, perfil avaliação de tecnologia e de programas em saúde. Brasília, DF. Email: flavia.tselias@gmail.com

${ }^{2}$ Departamento de Ciência e Tecnologia, Secretaria de Ciência, Tecnologia e Insumos Estratégicos, Ministério da Saúde
} 
Keywords: Technology Assessment, Biomedical, hospitals

RESUMEN: Objetivo: Analizar las características estructurales y procesos de trabajo después de dos años de la implementación de los centros de evaluación de la tecnología en hospitales en Brasil. Método: En este estudio se utilizó un cuestionario autoadministrado online que encuestó a gerentes de las veinticuatro centros que asistieron a la convocatoria pública de 2009. Resultados: un total de gerentes Veinte completó la encuesta. Catorce centros respondieron a la formalización de la sector en el hospital. Todos ellos reportaron dificultades en personal capacitado y los recursos financieros, los equipos fueron capaces de ofrecer cursos (dieciséis centros) y producir algunas evaluaciones como el asesoramiento de expertos científicos (doce centros). Diecinueve adoptó las directrices metodológicas de REBRATS. Conclusion: Se dio cuenta de que dos años de aplicación de estos centros no fueron suficientes para lograr las características estructurales y de procedimiento teniendo en cuenta la encuesta. La difusión de la cultura HTA en un país sin tradición como Brasil debe ser una acción permanente de la calidad de la asistencia sanitária.

Palabras llave: Evaluación de la Tecnología Biomédica, hospitales

\section{INTRODUÇÃO}

Avaliar a introdução de tecnologias de saúde e estabelecer uso apropriado de medicamentos e produtos requer processos críticos de avaliação das consequências clínicas, econômicas e sociais. Hospitais de ensino são locais privilegiados para formação de capacidades de avaliar tecnologias que devem ser utilizadas nas ações assistenciais, no ensino e na pesquisa (1). A implementação da avaliação de tecnologias realizadas em instituições de ensino e pesquisa voltadas para ações governamentais iniciou com formação da Rede Brasileira de Avaliação de Tecnologias em Saúde (REBRATS) em 2008 (2).

A institucionalização dos Núcleos de Avaliação de Tecnologias em Saúde (NATS) em Hospitais de Ensino ocorreu em anos posteriores como forma de envolver a gestores e técnicos de hospitais ligados ao Sistema Único de Saúde. A estratégia partiu do Grupo de Capacitação Profissional (3) com a Rede Sentinela da ANVISA. O objetivo foi envolver os participantes de cinco mestrados profissionais financiados pelo Departamento de Ciência e Tecnologia, do Ministério da Saúde e do curso de ensino à distância de saúde baseada em evidências promovido pelo Centro Cochrane do Brasil com o Hospital Sírio Libanês.

O Departamento de Ciência e Tecnologia, do Ministério da Saúde (DECIT/MS) e a AgênciaNacional de Vigilância Sanitária (ANVISA) realizaram edital público em parceria com a Organização Panamericana de Saúde (OPAS), para interessados em apresentar projetos para implantação de Núcleos de Avaliação de Tecnologias em Saúde (NATS) em Hospitais de 
Ensino. O valor global do financiamento foi de $\mathrm{R} \$ 600.000,00$ (seiscentos mil reais) que apoiou vinte e quatro projetos.

A implantação desses núcleos teve o objetivo de enraizar nos hospitais de ensino a cultura de avaliação de tecnologias, com ênfase na segurança de pacientes mediante uso racional de medicamentos e equipamentos, harmonização de diretrizes clínicas baseadas em evidências, racionalização da aquisição, retirada e uso de novas tecnologias. Essas práticas também deveriam influenciar na formação de pessoal e na identificação de inovações de produtos e serviços relevantes para efetividade da atenção à saúde no campo hospitalar (4).

Decorridos dois anos da criação destes núcleos, foram organizados seminários e reuniões nacionais da REBRATS para debater desafios para o desenvolvimento da área nos hospitais de ensino. O objetivo deste estudo foi analisar as características estruturais e os processos de trabalho dos núcleos de ATS em hospitais de ensino decorridos o período previsto no edital público de 2009.

\section{METODOLOGIA}

Foi aplicado um inquérito com os 24 Núcleos de Avaliação de Tecnologias em Saúde (NATS) criados, utilizando-se adaptação de questionário validado por Sub Grupo de Trabalho de avaliação e uso de tecnologias em serviços de saúde no âmbito do Mercado Comum do Sul (MERCOSUL), em 2011.

O questionário com questões objetivas e duas questões subjetivas foi disponibilizado para auto preenchimento utilizando-se o FormSUS, gerenciado pelo Departamento de Informática do SUS (DATASUS). Ficou disponível para preenchimento no período de 29/08/2011 a 14/10/2011, sendo divulgada quinzenalmente por meio de e-mail para os coordenadores dos núcleos. Disponibilizou-se telefone de contato para dúvidas de preenchimento.

A partir do questionário identificaram-se indicadores que correspondessem às dimensões relativas à estruturação dos núcleos, aos processos de trabalho implantados e aos resultados esperados. Quanto à estrutura, foram elencados os seguintes indicadores: presença de normas internas para formalizar o NATS, como portarias ou regimentos internos, infraestrutura física e de equipamentos; e quanto aos recursos humanos, foi verificada pelo número de pessoas, formação e tempo de dedicação ao NATS. Outro requisito analisado foi se os participantes tinham acesso às bases de dados, presença de orçamento próprio e outras formas de captação de recursos financeiros.

No que se refere ao processo de trabalho, foram elencados os seguintes indicadores: presença de instrumentos e fluxos operacionais instituído em manuais de procedimento, mecanismos para articulação com outras unidades hospitalares (gerenciamento de risco, pesquisa clínica, vigilância epidemiológica, auditoria e compras, infecção hospitalar) e formas de articulação com a gestão do SUS, como recebimento de demandas de secretarias estaduais e municipais. 
Quanto aos resultados, elegeu-se os seguintes indicadores: oferta de capacitações, estudos realizados por categoria, como: pareceres técnico científicos, revisões sistemáticas, avaliações econômicas, diretrizes clinicas revisadas ou elaboradas, assim como a origem das demandas de estudos.

Como questões subjetivas, incluiu-se a opinião dos coordenadores sobre as limitações para implantação das atividades e as contribuição dos núcleo para os processos internos do hospital.

No quadro 1 apresenta-se a lista de itens utilizados no questionário segundo dimensão analisada.

QUADRO 1: Lista de variáveis segundo dimensão, inquérito aplicado aos Núcleos de Avaliação de Tecnologias em Saúde em Hospitais de Ensino (NATS), Brasil 2011

\section{IDENTIFICAÇÃO: Nome da instituição, endereço, website, nome do informante, cargo} ou função, data de preenchimento.

\section{DIMENSÃO ESTRUTURAL}

- Documento de formalização do núcleo. Área/departamento da instituição o NATS está subordinado. Local físico específico para funcionamento

- Número de computadores com acesso à internet banda larga e assinatura de bases de dados científicas

- Presença de equipe técnica capaz de ler e entender documentos nos idiomas Espanhol, Português, Inglês ou outro. Número de profissionais, quantidades de hora/trabalho por semana dedicadas à execução da ATS, por tipo de graduação. Quantidade de profissionais com formação específica em ATS

- Existência de orçamento específico da Instituição para a manutenção do Núcleo. Participação em algum outro edital de pesquisas no campo da ATS financiado pelo Ministério da Saúde em anos posteriores da criação do NATS. Existência de algum outro financiamento externo nos anos 2010 e 2011

PROCESSO DE TRABALHO 
a) Listagem de serviços do hospital em que o NATS desenvolve atividades conjuntas.

- Consultoria sobre aquisição/utilização de tecnologias em saúde

- Avaliação da eficácia, eficiência e efetividade dos processos em saúde

- Avaliação da introdução de novos procedimentos organizacionais

- Consultoria sobre planos estratégicos das tecnologias em saúde

- Avaliação da segurança de novas tecnologias

- Programa de gerenciamento de riscos

- Elaboração de Diretrizes e Protocolos Clínicos

- Avaliação de protocolos em vigilância epidemiológica

- Auditoria Clínica

- Consultoria sobre pesquisa experimental

- Pesquisa e Desenvolvimento

- Programas educacionais (capacitação) e Eventos

- Atividade de monitoramento do horizonte

tecnológico b) Adoção de procedimentos normativos

- Existência de fluxo formalizado para atender as demandas internas

- Existência de fluxo formalizado para atender as demandas externas ao hospital

- Tipo de tecnologias avaliadas com mais frequência

c) Foco da avaliação: Eficácia, Efetividade, Custo-efetividade (ou Custoutilidade),Produtividade Técnica,Impacto Orçamentário,Impacto Organizacional, questões Éticas, Perspectivas dos pacientes, Satisfação de pacientes, Outras.

- Existência de critérios para definição de prioridades das demandas de ATS

- Atores consultados para ajudar a estabelecer prioridades

- Utilização das diretrizes metodológicas da REBRATS

- Presença de abordagem de custos e análises econômicas nas avaliações de tecnologias de saúde

- Modelos de Avaliação Econômica que são usualmente executadas: Análise de custominimização (ACM), Análise de custo-efetividade (ACE), Análise de custo-benefício (ACB)

- Parcerias com alguma outra instituição para a execução dos estudos de ATS.

\section{RESULTADOS ESPERADOS}

- Atividades de capacitação, nome do curso, ano e o número de alunos

- Número de estudos produzidos por tipo de estudo e classificação segundo a CID 10

- Origem da demanda dos estudos produzidos pelo NATS

- Estudos produzidos segundo tipo de contribuição: incorporação de novas tecnologias, retirada de tecnologias obsoletas, elaboração de guias de protocolos/diretrizes clínicos, Outros

- Principais limitações encontradas por sua organização na execução de atividades de ATS

- Contribuição do NATS para sua Instituição

A sistematização das respostas foi obtida a partir dos relatórios gerados pelo FormSUS e a análise se deu utilizando-se frequência simples das variáveis com maior completude de respostas. 


\section{RESULTADOS}

A taxa de resposta da aplicação dos questionários foi de $83 \%$, correspondente a 20 instituições entre as 24 incluídas na pesquisa.

Em relação à dimensão estrutural, 14 núcleos têm sua criação formalizada, dos quais 12 forneceram instrumento comprobatório. Ao menos 19 NATS possuíam local físico específico para seu funcionamento e ao menos um computador com internet banda larga. Apenas dois núcleos têm orçamento anual (Tabela 1).

\begin{tabular}{|c|c|c|c|}
\hline Indicador & Presença & Ausência & Não respondida \\
\hline \multicolumn{4}{|c|}{ Dimensão Estrutural } \\
\hline Institucionalização $(n=24)$ & $58 \%(14)$ & $\begin{array}{l}25 \% \\
(06) \\
\end{array}$ & $17 \%(04)$ \\
\hline Regimento/Portaria $(n=24)$ & $55 \%(11)^{*}$ & $\begin{array}{l}25 \% \\
(06) \\
\end{array}$ & $17 \%(04)$ \\
\hline Profissionais dedicados à ATS $(\mathrm{n}=20)$ & $100 \%(20)$ & & \\
\hline 01 a 03 profissionais & 4 & & \\
\hline 04 a 06 profissionais & 9 & & \\
\hline $\begin{array}{r}07 \text { a } 10 \text { profissionais } \\
\text { Acima de } 10 \\
\end{array}$ & $\begin{array}{l}1 \\
6\end{array}$ & & \\
\hline \multicolumn{4}{|l|}{ Formação profissional $(\mathrm{n}=20)$} \\
\hline Médico & $100 \%(20)$ & & \\
\hline Engenheiro & $30 \%(6)$ & & \\
\hline Farmacêutico & $65 \%(13)$ & & \\
\hline Enfermeiro & $60 \%(12)$ & & \\
\hline Acesso a bases de dados $(n=20)$ & $95 \%(19)$ & & $5 \%(1)$ \\
\hline $\begin{array}{r}\text { EMBASE } \\
\text { MEDLINE/PUBMED } \\
\text { Cochrane } \\
\text { INAHTA } \\
\text { LILACS } \\
\text { Outras }\end{array}$ & $\begin{array}{c}42 \%(8 / 19) \\
100 \%(19 / 19) \\
94 \%(18 / 19) \\
68 \%(13 / 19) \\
94 \%(18 / 19) \\
26 \%(5 / 19) \\
\end{array}$ & & \\
\hline Orçamento anual próprio $(n=20)$ & $10 \%(02)$ & $\begin{array}{l}90 \% \\
(18) \\
\end{array}$ & \\
\hline \multicolumn{4}{|c|}{$\begin{aligned} \text { Processo de trabalho (continua) } \\
\end{aligned}$} \\
\hline $\begin{array}{l}\text { Instrumentos e/ou fluxos operacionais } \\
\text { instituído em manuais de procedimento. } \\
(\mathrm{n}=20)\end{array}$ & $\begin{array}{l}35 \%(7) \\
15 \%(3)\end{array}$ & $\begin{array}{l}65 \% \\
(13) \\
85 \% \\
(17)\end{array}$ & \\
\hline
\end{tabular}




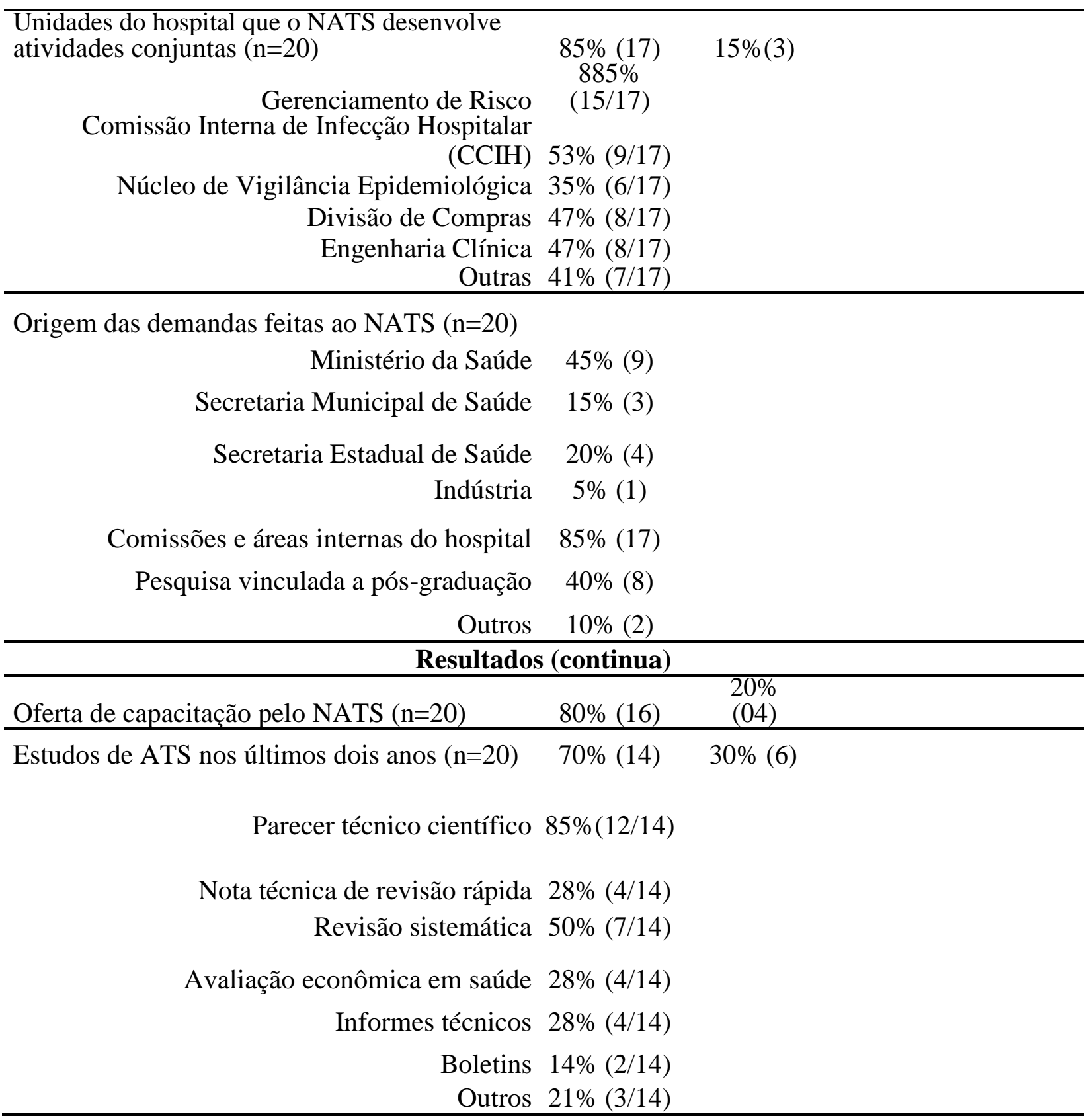

Fonte: Elaboração Própria. Nota (*) Dois NATS não anexaram o documento comprobatório

O acesso livre a bases de dados da literatura científica foi relatado pela maioria dos núcleos, mas o acesso à bases pagas, como o Embase, foi relatada por 8 núcleos (Tabela 1).

Os dados referentes à equipe do NATS demonstram que 9 núcleos dispõem de 4 a 6 profissionais, exercendo atividades em dedicação exclusiva ou parcial.

A articulação com outras unidades do hospital foi relatada por 16 núcleos, destacando o setor de gerenciamento de risco, seguido da comissão de infecção hospitalar. As demandas internas (Tabela 1) foram provenientes de comissões hospitalares e as externas são de secretarias estaduais e municipais de saúde e do Ministério da Saúde. 
A oferta de capacitação pelo NATS foi um tipo de atividade bastante frequente em 16 núcleos. A atividade relatada incluía desde seminários a cursos de média duração.

O foco da avaliação em 16 instituições foi direcionado a estudos de eficácia, seguidos por estudos de efetividade (15 instituições) e de impacto orçamentário, custo-efetividade (ou custo-utilidade) e segurança (12 instituições). Todos os 20 NATS declararam utilizar como fonte de informação nos pareceres técnicos científicos as revisões sistemáticas ou os informes de ATS de agências internacionais. Apenas 13 declararam realizar revisões sistemáticas de estudos primários. Ao menos 19 NATS afirmaram utilizar as diretrizes metodológicas da REBRATS, publicadas pelo

Ministério da Saúde para o desenvolvimento dos seus estudos.

Os estudos desenvolvidos pelos núcleos estavam distribuídos tanto em doenças crônicas, como em doenças infecciosas, parasitárias e causas perinatais segundo grande área da Classificação internacional de doença (Tabela 2).

Tabela 2: Estudos de ATS desenvolvidos pelos NATS, segundo CID 10, 2011.

\begin{tabular}{lrr} 
& Qtd & Qtd \\
\hline \multicolumn{1}{c}{ Grande área CID 10 } & $\mathbf{9}$ & $\mathbf{4 5} \%$ \\
\hline Algumas doenças infecciosas e parasitárias & $\mathbf{8}$ & $\mathbf{4 0} \%$ \\
Neoplasias & $\mathbf{5}$ & $\mathbf{2 5} \%$ \\
Doenças do sangue e dos órgãos hematopoéticos e alguns & $\mathbf{8}$ & $\mathbf{4 0} \%$ \\
transtornos imunitários & $\mathbf{2}$ & $\mathbf{1 0} \%$ \\
Doenças endócrinas, nutricionais e metabólicas & $\mathbf{4}$ & $\mathbf{2 0} \%$ \\
Transtornos mentais e comportamentais & $\mathbf{1}$ & $\mathbf{5 \%}$ \\
Doenças do sistema nervoso Doenças do olho e anexos Doenças do aparelho & $\mathbf{8}$ & $\mathbf{4 0} \%$ \\
circulatório Doenças do aparelho respiratório & $\mathbf{4}$ & $\mathbf{2 0} \%$ \\
Doenças do aparelho digestivo & $\mathbf{4}$ & $\mathbf{2 0} \%$ \\
Doenças da pele e do tecido subcutâneo Doenças do sistema osteomuscular e do & $\mathbf{1}$ & $\mathbf{5 \%}$ \\
tecido conjuntivo & $\mathbf{3}$ & $\mathbf{1 5 \%}$ \\
Doenças do aparelho geniturinário & & $\mathbf{4 0}$ \\
Gravidez, parto e puerpério & $\mathbf{4}$ & $\mathbf{2 0}$ \\
Algumas afecções originadas no período perinatal & $\mathbf{4}$ & $\mathbf{2 0} \%$ \\
Malformações congênitas, deformidades e anomalias cromossômicas & $\mathbf{3}$ & $\mathbf{1 5 \%}$ \\
Fonte: Questionário aplicado aos NATS. Elaboração Própria & $\mathbf{2}$ & $\mathbf{1 0} \%$ \\
Or equipamentos biomédicos foram raramente avaliados por 10 NATS, e os & & \\
protocolos clínicos & &
\end{tabular}


Foram regularmente avaliados por cerca de 12 núcleos. Ao menos 6 núcleos relataram avaliar medicamentos. Em contraposição, outros 6 núcleos relataram avaliar raramente esse tipo de tecnologia (Gráfico 1).

Gráfico 1: Frequência de tecnologias avaliadas, segundo o tipo de tecnologia, inquérito com NATS, 2011.

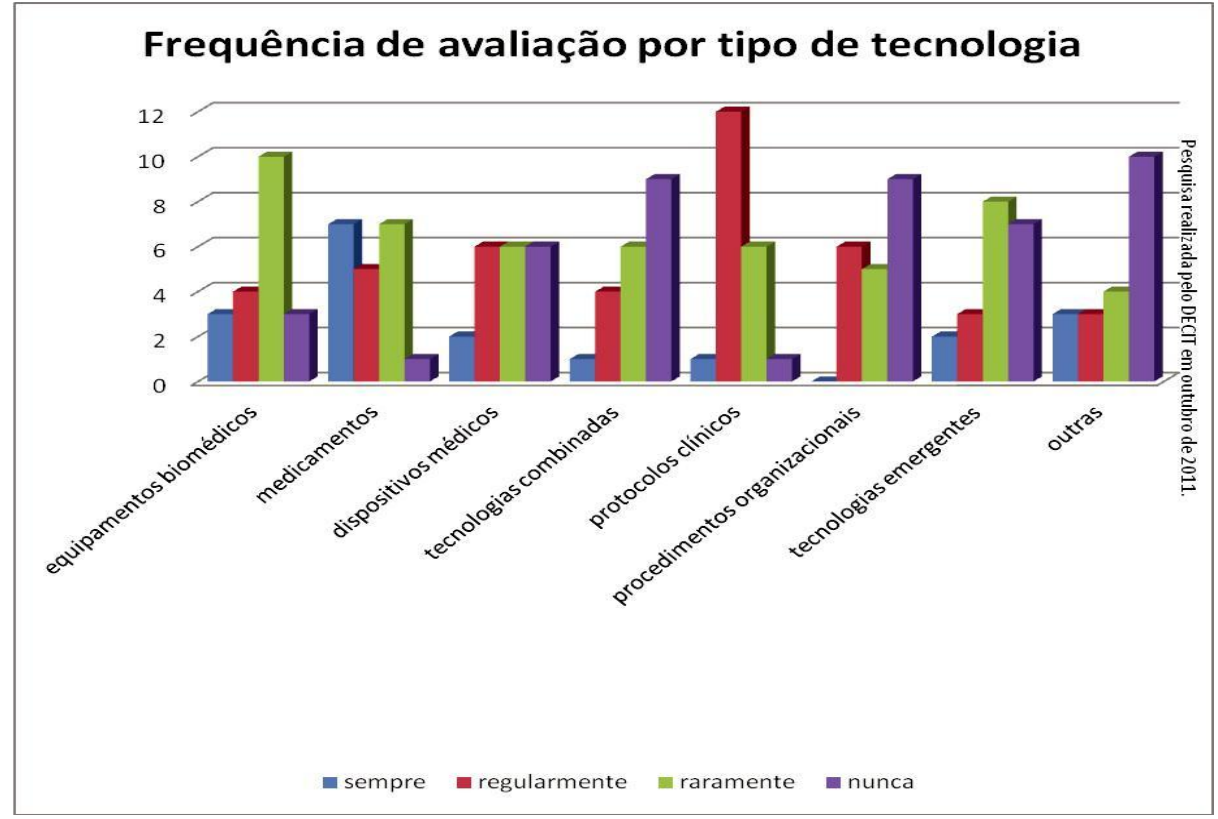

Fonte: Questionário aplicado aos NATS. Elaboração Própria

Em relação às principais atividades executadas pela instituição, 17 NATS relataram que elaboraram diretrizes técnicas e protocolos clínicos. Apenas 12 relataram que entre suas atividades estavam direcionadas a orientar a aquisição/utilização de tecnologias, a avaliação da eficácia, eficiência e efetividade dos processos em saúde e programa de gerenciamento de riscos.

Duas limitações estruturais para executar as atividades de ATS tiveram a mesma frequência de resposta, ou seja, 16 núcleos relataram dificuldade de dispor de pessoal capacitado e de recursos financeiros (Gráfico 2).

Gráfico 2: Dificuldades relatadas pelos NATS para execução de ATS, inquérito 2011.

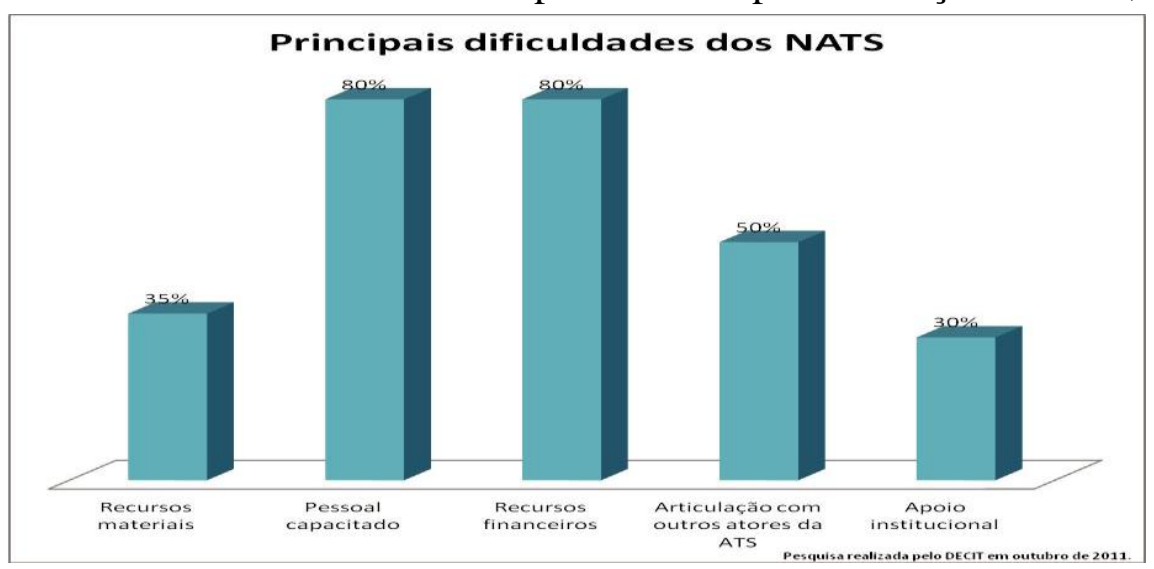

Fonte: Questionário aplicado aos NATS.Elaboração Própria 
A opinião dos 20 respondentes foi diversificada quanto a questão subjetiva sobre as contribuições da criação dos núcleos para influenciar os processos e ações assistenciais internos ao hospital. Ao menos dois núcleos observaram que o núcleo permaneceu inoperante. Os demais núcleos relataram que foi possível realizar ações de capacitação de profissionais e disseminar informações sobre as possíveis aplicações da ATS. Apenas um núcleo relatou ter obtido apoio institucional para novos recursos financeiros. A minoria dos respondentes citou haver influências em processos assistenciais internos ao hospital, mesmo tendo afirmado receber demandas de áreas internas. Em relação as contribuições em processos de atuação externos ao hospital, dois núcleos citaram ter estabelecido relações formais com o Judiciário e com outras instituições da REBRATS. Dois citaram contribuir com decisões do Ministério da Saúde.

\section{DISCUSSÃO}

Percebeu-se com os resultados do inquérito de 2011, que a implementação de vinte núcleos de avaliação de tecnologias em saúde em hospitais de ensino permanecia inconclusa após dois anos de execução do projeto. $\mathrm{O}$ fato de ter normas internas formalizando o NATS, o que aconteceu com 14 deles, não foi fator favorável para garantir a execução das ações de ATS.

O processo de trabalho pôde ser destacado pelo número de núcleos que relataram ter instrumentos e fluxos operacionais instituídos nos manuais de procedimento, sendo sete destinados a ordenar demandas internas e três para atender demandas externas.

No entanto, para aqueles que conseguiram alguma estruturação, mesmo tendo relatado dificuldade de pessoal capacitado e recursos financeiros, conseguiram ofertar atividades de capacitação e produzir algumas avaliações, na sua maioria, pareceres técnicos científicos. Esses tipos de estudos podem ser considerados mini- health tecnology assessment (MiniHTA), muito empregados em hospitais (5).

Não foi possível realizar visitas locais para aplicação do questionário. Esse fato pode ter gerado erros de interpretação nas questões, como as divergências declaradas pelos respondedores ao se observar respostas incongruentes para questões semelhantes. O instrumento informatizado embora tenha vantagens, limitou a forma de estruturar as perguntas e a sistematização de respostas, não sendo possível realizar análises estatísticas para associação de variáveis.

Contudo, esse achado não coaduna com as respostas à questão subjetiva sobre contribuições dos núcleos nos processos de decisão internos ao hospital. Esse fato abre espaço para investigação de barreiras que explicam que a maioria dos coordenadores relataram poucas influencias em processos internos.

Em que pese as limitações encontradas, de modo geral, os resultados obtidos corroboram com a literatura internacional (5). A utilização da ATS no campo hospitalar se encontra em fase inicial. O cenário é bastante propício para sua aplicação (6), haja vista o contexto tecnológico que esses serviços albergam, seja nas diversas categorias de tecnologias, inclusive nos procedimentos assistenciais, técnicas de acolhimento e acompanhamento de pacientes. 
Em 2006, a Sociedade Internacional de ATS (HTAi) estabeleceu um Subgrupo dedicado à aplicação da lógica de ferramentas de ATS na dimensão hospitalar. Essa ação internacional mostra como a adoção de novos métodos em ATS na dimensão da gestão hospitalar tem ganhado importância nos últimos encontros sobre o tema (6).

A difusão do uso da lógica de ATS em hospitais e outros estabelecimentos de assistência à saúde pode ser considerada como uma forma de administradores hospitalares responder a três diferentes pressões. A primeira diz respeito ao aumento da "pressão" que as autoridades de saúde estão exercendo aos indivíduos das organizações de saúde com vistas à melhoria do nível de eficiência e eficácia microeconômica como chave para aperfeiçoar a eficiência de todo o sistema macroeconômico (6).

A segunda está relacionada com o reconhecimento progressivo da importância do fator "contexto". Oportunidades e vantagens que podem ser tiradas a partir do uso de tecnologias em saúde no campo da área médica variam de acordo com a disponibilidade de recursos e competências em cada organização de saúde. Por esta razão, se a decisão deve ser tomada em um nível organizacional, evidências e dados devem ser também coletados e analisados no contexto organizacional (6),(7).

Uma terceira pressão está relacionada com a difusão da cultura da medicina baseada em evidências. Políticos, gestores hospitalares, médicos e outros profissionais estão cada vez mais inseridos na responsabilidade em adotar os conceitos de ATS nos seus serviços (6), (7).

\section{CONCLUSÃO}

O diagnóstico desenvolvido com os hospitais de ensino que iniciaram a criação de núcleos de ATS identificou que os dois anos de implantação, não foram suficientes para obter a organicidade de estrutura e processos destinados a estabelecer uma ação de ATS para dentro das atividades gestoras do hospital.

A principal característica estrutural positiva foi a formalização da área no âmbito do hospital sem contudo, garantir execução completa das ações de ATS previstas, nem tão pouco, possibilitar orçamento contínuo. Embora tenham relatado dificuldades em obter pessoal capacitado e em garantir recursos financeiros, a maioria dos núcleos conseguiu ofertar cursos e produzir algumas avaliações como os pareceres técnicos científicos. As diretrizes metodológicas da REBRATS publicadas pelo Ministério da Saúde parecem ter sido utilizadas pela maioria dos núcleos.

A Comissão Nacional de Incorporação de Tecnologias no Sistema Único de Saúde, órgão colegiado do Ministério a Saúde, reestruturado em 2011, também se apoia nas experiências dos hospitais para deliberar sobre tecnologias a serem financiadas pelo sistema. O Departamento de Gestão e Incorporação de Tecnologias, também membro da REBRATS, exerce a secretaria executiva da comissão e atua para implantar novos núcleos para inserção na rede;

A perspectiva é de continuidade, pois a estratégia de fortalecer a ATS em hospitais se torna fundamental para melhoria da qualidade da assistência e da segurança de pacientes. Em 2012, foi criado o grupo de serviços de saúde na REBRATS, responsável por estimular a aplicação da ATS em órgãos do SUS, como secretarias de saúde e hospitais. A difusão da cultura de ATS em país ISSN 1982-8829 
sem tradição como o Brasil deve ser uma ação permanente nas instituições que colaboram para um sistema de saúde de qualidade.

\section{REFERÊNCIAS BIBLIOGRÁFICAS}

1.GARRIDO, M V ET AL. Health Technology Assessment in Europe - overview of producers. In Garrido, M V; Kristensen Finn B; Nielsen, CP, Busse, R. Health Technology Assessment and health policy making in Europe. Current status, challenges and potential. Eunehta/European Observatory/ WHO, 2008. Observatory Studies n. 14.

2. BANTA, R \& ALMEIDA, R T. The development of Health technology assessment in Brazil. Int. J. of Technology Assessment in Health Care 25. Supplement 1, 2009.

3. BRASIL. MINISTÉRIO DA SAÚDE. Portaria n. 2.915/GM, de 12 de dezembro de 2011. Institui a Rede Brasileira de Avaliação de Tecnologias em Saúde (REBRATS). 201. www.rebrats. gov.br

4. Departamento de Ciência e Tecnologia, Secretaria de Ciência, Tecnologia e Insumos Estratégicos, Ministério da Saúde - DECIT/SCTIE/MS. Consolidação da área de avaliação de tecnologias em saúde no Brasil. Rev Saúde Pública 2010; 44(2): 381-3. http://www.scielo.br/pdf/ rsp/v44n2/22.pdf

5. MARTELLIA, N; LELONGA, AS; PROGNONA, P ; PINEAUA, J Hospital-Based Health Technology Assessment for Innovative Medical Devices in University Hospitals and the role of Hospital Pharmacists: Learning from International Experience International Journal of Technology Assessment in Health Care, 29(2):185-191 April 2013

\section{HOSPITAL BASED HEALTH TECHNOLOGY ASSESSMENT SUB-INTEREST}

GROUP. Hospital Based Health Technology Assessment World-Wide Survey. Disponível em http://www.htai.org/fileadmin/HTAi_Files/ISG/HospitalBasedHTA/2008Files/ HospitalBasedHTAISGSurveyReport.pdf [Acesso em 16. fev 2015].

7. FAVARETTI C, CICCHETTI A, GUARRERA G, MARCHETTI M; RICCIARDI W (2009). Health technology assessment in Italy. International Journal of Technology Assessment in Health Care, 25:127-133. 